\title{
Comunicação
}

[Communication]

\section{Parasitoides de Ornidia obesa Fabricius (Diptera: Syrphidae) coletados em fezes de galinha no Brasil}

[Parasitoids of Ornidia obesa Fabricius (Diptera: Syrphidae) collected in chicken feces in Brazil]

\author{
C.H. Marchiori, L.A. Bessa, A.L. Ribeiro
}

Instituto Federal de Educação - Ciência e Tecnologia Goiano- IF Goiano - Goiânia, Goiás

A alta densidade de aves em granjas causa um grande acúmulo de esterco, um excelente substrato para a proliferação de moscas. Para se obter um controle satisfatório destes insetos, um programa de manejo deve integrar métodos culturais, químicos e biológicos. Os agentes de controle biológico terão maior chance de serem bem-sucedidos se forem mais adaptados à região, à época do ano e ao tipo de esterco do local (Costa et al., 2004).

Parasitoides são importantes reguladores populacionais de insetos e se destacam como principal grupo de inimigos naturais em sistemas agrícolas. Estão dispersos em inúmeras famílias de insetos, e sua adaptação ao modo de vida parasítico é mais diversa e abundante em Hymenoptera (Panizzi e Parra, 2009). Os parasitoides são agentes responsáveis pela redução de moscas que proliferam em esterco, cadáveres e carcaças de animais.

A espécie Ornidia obesa Fabricius (Diptera: Syrphidae) é extensivamente distribuída nas Américas e disseminou-se amplamente no Velho Mundo no século XIX por meio do comércio (Morales e Köhler, 2004). Os adultos de $O$. obesa abrigam bactérias de importância para a saúde pública (Podlipaev e Naumov, 2000; Morales e Köhler, 2004).

Spalangia cameroni Perkins (Hymenoptera: Pteromalidae) é considerado um parasitoide solitário de pupários numerosos Diptera nas famílias Anthomyiidae, Muscidae, Otitidae e Sarcophagidae (Bai e Sankaran, 1977;
Blume, 1984; Sereno e Neves, 1993). A espécie Pachycrepoideus vindemmiae (Rondani) (Hymenoptera: Pteromalidae) é considerada um parasitoide solitário de numerosos Diptera nas famílias Anthomyiidae, Calliphoridae, Muscidae, Sarcophagidae, Tachinidae, Tephritidae e outras. Esta espécie apresenta ampla distribuição geográfica, sendo também encontrada na América do Norte e África (Hanson e Gauld, 1995). O objetivo deste trabalho foi relatar os parasitoides $O$. obesa coletados no Brasil.

O experimento foi realizado na granja do CEFET-UNED, Morrinhos, em Goiás. As fezes coletadas originaram-se de 80 aves de linhagem comercial. A granja é composta por dois galpões de $15 \mathrm{~m}$ de comprimento por $5 \mathrm{~m}$ de largura e $5 \mathrm{~m}$ de altura. $\mathrm{O}$ galpão é formado por duas fileiras de gaiolas construídas de armação de metal contendo duas aves por gaiola. As fileiras com as gaiolas ficam separadas por um corredor de cimento de $2 \mathrm{~m}$ de largura, e estas ficam suspensas do chão a $50 \mathrm{~cm}$ de altura. O piso abaixo das gaiolas e ao redor é constituído por terra. As fezes acumuladas sob as gaiolas variavam de umidade, de consistência pastosa ou firme. Em um galpão, as fezes frescas foram coletadas imediatamente após a emissão e colocadas em quatro bacias de $30 \mathrm{~cm}$ de diâmetro por $12 \mathrm{~cm}$ de altura, as quais foram deixadas no próprio ambiente, em local seco. As fezes permaneceram no galpão por 15 dias; posteriormente, as bacias foram retiradas $\mathrm{e}$ levadas para o laboratório para a extração das pupas pelo método da flotação. As pupas foram retiradas com o auxílio de uma peneira, contadas

Recebido em 4 de fevereiro de 2010

Aceito em 6 de abril de 2011

E-mail:chmarchiori@yahoo.com.br 
e individualizadas em cápsulas de gelatina (número 00) até a emergência dos dípteros e/ou dos seus parasitoides. Os parasitoides e os dípteros emergidos foram identificados com auxílio de um microscópio estereoscópio e, posteriormente, conservados em álcool $70 \%$.

A porcentagem de parasitismo foi calculada dividindo-se o número de pupas parasitadas pelo número total de pupas coletadas, multiplicandose o resultado por cem.

No período de abril a dezembro de 2007, coletaram-se 39 exemplares de O. obesa, dos quais emergiram dois exemplares do parasitoide $P$. vindemmiae e dois exemplares de $S$. cameroni. A porcentagem de parasitismo foi de $5,1 \%$ tanto para $P$. vindemmiae quanto para $S$. cameroni, a porcentagem total de parasitismo foi de $10,3 \%$.

No Brasil, a espécie $P$. vindemmiae foi encontrada parasitada com Chrysomya albiceps (Wiedemann) (Diptera: Calliphoridae), Fannia pusio (Wiedemann) (Diptera: Fanniidae), Oxysarcodexia thornax (Walker) (Diptera:
Sarcophagidae), Peckia chrysostoma (Diptera: Sarcophagidae), Sarcodexia sp. (Diptera: Sarcophagidae), Sarcophagula sp. (Diptera: Sarcophagidae) e Poecilosomella sp. (Diptera: Sphaeroceridae) (Marchiori et al., 2002).

No Brasil, S. cameroni foi encontrada parasitando Chrysomya putoria (Wiedemann) (Diptera: Calliphoridae), Fannia trimaculata (Stein) (Diptera: Fanniidae) Musca domestica L. (Diptera: Muscidae), Muscina stabulans (Fallèn) (Diptera: Muscidae), Stomoxys calcitrans (L.) em fezes de galinha (Costa et al. 1992; Sereno e Neves, 1993; Marchiori et al. 2003).

Microimenópteros parasitoides da família Pteromalidae são naturalmente coletados emergindo de pupários de dípteros que proliferam no esterco de aves, e algumas espécies podem ser utilizadas como controladores biológicos (Sereno e Neves, 1993).

Palavras-chave: Hymenoptera, fezes de galinha, inimigo natural, controle biológico, Goiás

\begin{abstract}
This study reports the occurrence of Pachycrepoideus vindemmiae (Rondani) (Hymenoptera: Pteromalidae) and Spalangia cameroni Perkins (Hymenoptera: Pteromalidae) as parasitoids of Ornidia obesa Fabricius (Diptera: Syrphidae) in poultry farms in Morrinhos, Goiás, state Brazil. Samples of chicken feces were collected at two weeks intervals and taken to the laboratory. The pupae were extracted by water flotation. Each pupa was placed in capsules of gelatin until the emergence of dipterous or their parasitoids. The parasitism percentage of $\mathrm{P}$. vindemmiae and $\mathrm{S}$. cameroni was $5.1 \%$.
\end{abstract}

Keywords: Hymenoptera, chicken dung, natural enemy, biocontrol, Goiás

\section{REFERÊNCIAS}

BAI, M.G.; SANKARAN, T. Parasites, predators and other arthropods associated with Musca domestica and other flies breeding in bovine manure. Entomophaga, v.22, p.163-167, 1977.

BLUME, R.R. Parasites of Diptera associated with bovine droppings on a pasture in east Central Texas. South. Entomol., v.11, p.215-222, 1984.
COSTA, V.A.; BERTI FILHO, E.; SILVEIRA NETO, S. Parasitoides (Hymenoptera: Chalcidoidea) de moscas sinantrópicas (Diptera: Muscidae) em aviários de Echaporã, SP. Arq. Inst. Biol., v.71, p.203-209, 2004.

COSTA, V.A.; GUIMARÃES, J.H.; TUCCI, E.C. et al. Parasitoides associados a dípteros sinantrópicos de granjas de aves de postura no estado de São Paulo, Brasil. Rev. Bras. Parasitol. Vet., v.1, p.55-59, 1992. 
HANSON, P.E.; GAULD, I.D. The Hymenoptera of Costa Rica. Oxford: University of Oxford, 1995. 893p.

MARCHIORI, C.H.; PEREIRA, L.A.; SILVA FILHO, O.M. et al. Pachycrepoideus vindemmiae Rondani (Hymenoptera: Pteromalidae) as parasitoid of Diptera, in Brazil. Arq. Bras. Med. Vet. Zootec., v.54, p.665-667, 2002.

MARCHIORI, C.H.; SILVA FILHO, O.M.; BORGES, M.P. et al. Parasitoides de Musca domestica L. (Diptera: Muscidae) de Itumbiara, Goiás, Brasil. Rev. Patol. Trop., v.32, p.263-266, 2003.

MORALES, M.N.; KÖHLER, A. Ornidia Lepeletier e Serville, 1828 (Diptera, Syrphidae) no estado do Rio Grande do Sul, Brasil: distribuição e preferência floral. Rev. Bras. Zootec., v.6, p.93-102, 2004.
PANIZZI, A.R.; PARRA, J.P. Bioecologia $e$ nutrição de insetos. Brasília: Embrapa, 2009. $1163 \mathrm{p}$.

PODLIPAEV, S.A.; NAUMO, V.A.D. Colonies of trypanosomatids on agar plates: the tool for differentiation of the species and isolates. Protistology, v.1, p.113-119, 2000.

SERENO, F.T.P.S; NEVES, D. Microimenópteros (Pteromalidae) parasitoides de Diptera (Muscidae, Otitidae) em uma granja de bovinos em Igarapé, estado de Minas Gerais, Brasil. Rev. Bras. Entomol., v.37, p.563-567 1993. 